\title{
Comparative Performance Analysis of Hamming, Hanning and Blackman Window
}

\author{
Prajoy Podder \\ Department of ECE \\ Khulna University of \\ Engineering \& Technology \\ Khulna-9203, Bangladesh
}

\author{
Tanvir Zaman Khan
Department of ECE
Khulna University of
Engineering \& Technology
Khulna-9203, Bangladesh \\ Tanvir Zaman Khan
Department of ECE
Khulna University of
Engineering \& Technology
Khulna-9203, Bangladesh \\ Tanvir Zaman Khan
Department of ECE
Khulna University of
Engineering \& Technology
Khulna-9203, Bangladesh \\ Tanvir Zaman Khan
Department of ECE
Khulna University of
Engineering \& Technology
Khulna-9203, Bangladesh \\ Tanvir Zaman Khan
Department of ECE
Khulna University of
Engineering \& Technology
Khulna-9203, Bangladesh
}

M. Muktadir Rahman

Department of ECE

Khulna University of

Engineering \& Technology

Khulna-9203, Bangladesh

\author{
Mamdudul Haque Khan \\ Department of ECE \\ Khulna University of \\ Engineering \& Technology \\ Khulna-9203, Bangladesh
}

\begin{abstract}
In the emerging field of medical image processing, computer vision, pattern recognition and other digital signal processing applications, window technique is vastly used. A window function is a mathematical function that is zero-valued outside of some chosen interval. When another function is multiplied by a window function, the product is also zero-valued outside the interval. In this paper, the performance of Hamming, Hanning and Blackman window have been mainly compared considering their magnitude response, phase response, equivalent noise bandwidth, sidelobe transition width, response in time and frequency domain using MATLAB simulation. To observe the responses, a FIR filter of low pass, high pass, band pass and band stop type have been designed and encountered them with each parameters stated above. The results that have been found is as same as its to be as stated in the theory. Comparing simulation results of different window, this paper has found Blackman window with best performance among them which is also expected from the theory. These windows have also been encountered with speech signal using MATLAB simulation and found the same expected result.
\end{abstract}

\section{Keywords}

FIR filter, Impulse response, Magnitude response, Equivalent noise bandwidth.

\section{INTRODUCTION}

Filter is a kind of electronic device without which the whole communication system and signal processing whether digital or analog will go back to the Dark Age. Filter defines, contains and provides identity to each user and thus ensures that the expected signal has been forwarded to the expected direction. In signal processing, a filter is a device or process that removes from the unwanted component of the signal. Different forms of filters are used for different purposes. Low pass filters are used to pass the low frequency band. Band pass filters are used to pass a suitable frequency band that is required for desired applications. Digital filters can be classified into two categories and they are the Finite Impulse Response (FIR) filter and Infinite Impulse Response (IIR) filter [1][2]. In the FIR system, the impulse response is of finite duration, this means that it has a finite number of nonzero terms. On the other hand, The IIR system has an infinite number of nonzero terms. This means its impulse response is of infinite duration. While implementation, FIR filter needs no feedback. FIR filter is not a recursive filter. Because of this reason, the structure of FIR filter is much more simpler than compared to the IIR filter [3][4][5]. For designing the FIR filter, the analog filter is first built by active or passive elements. The analog filter is then diagrammed suitably into digital domain using the required IIR filter. Then by applying proper method, generally applying Fourier series method ,Frequency sampling method or Window method, the FIR filter can be obtained[4][6][7][8]. There are some problems included in the implementation of FIR filters using Fourier series method. The abrupt truncation of the Fourier series results in oscillations in the pass band and stop band. These oscillations are due to slow convergence of the Fourier series, particularly near the points of discontinuity. These problems can be solved by using an appropriate window function. Window methods are widely used. In this paper, among all the windows, Blackman window and the Hamming window are discussed for designing the FIR filter. Here, the gain responses are observed by designing FIR low pass, high pass, band pass, band stop filter using Hamming, Hanning and Blackman window. There are certain benefits of Blackman window over the Hamming and Hanning window [8][9][10]. A comparative discussion is presented in this paper along with the appropriate simulation of FIR filter by using Blackman window and the Hamming and Hanning window.

\section{FIR FILTER}

The response of the FIR filter depends only on the present and past input samples. There are some forms of FIR filter. They are [9][10][11][12][13]:

\section{High pass filter \\ 2. Low pass filter \\ 3. Band pass filter \\ 4. Band stop filter \\ 5. All pass filter}

The benefits of FIR filter over IIR filter are given below [4][6][14][17][18]: 
1. FIR filters are stable.

2. They can have an exact linear phase.

3. They can be realized efficiently in hardware.

4. The filter start-up transients have finite duration.

5. Simple extensions to multi rate and adaptive filters

6. FIR filter follows the procedures of Kronecker delta rule which says the impulse response will be zero after a finite time. The Kronecker delta is defined by,

$\delta_{i j}=\left\{\begin{array}{ll}1, & \text { if } i=j \\ 0, & \text { if } i \neq j\end{array}\right\}$

Now eqn. (2.1) is valid only for two variables i.e. $i$ and $j$. If there one variable exists, the Kronecker delta becomes,

$\delta_{i}=\left\{\begin{array}{ll}1, & \text { if } i=0 \\ 0, & \text { if } i \neq 0\end{array}\right\}$

\section{DEFINITION OF FIR FILTER}

Now For a discrete-time FIR filter, the output is a weighted sum of the current and a finite number of previous values of the input. The operation is described by the following equation, which defines the output sequence $y[n]$ in terms of its input sequence $\mathrm{x}[\mathrm{n}]$ :

$$
\begin{aligned}
y(n) & =b_{0} x[n]+b_{1} x[n-1]+\ldots \ldots+b_{N} x[n-N] \\
& =\sum_{i=1}^{n} b_{i} x[n-i]
\end{aligned}
$$

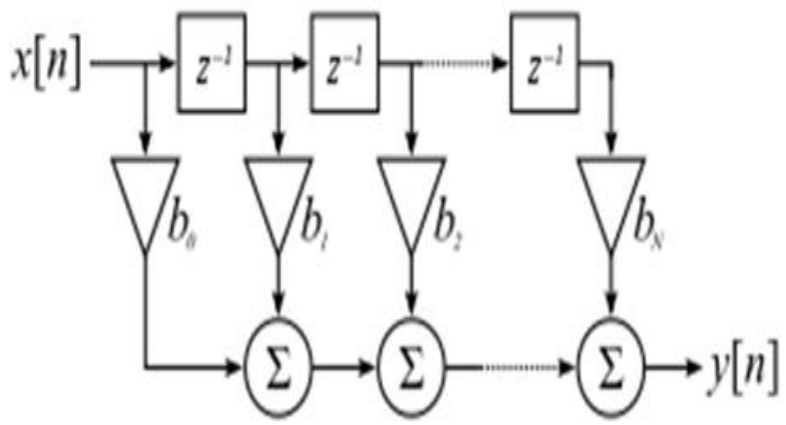

Fig.1: A discrete-time FIR filter of order N. The top part is an $\mathrm{N}$-stage delay line with $\mathrm{N}+1$ taps.

\section{WINDOW TECHNIQUE}

Window technique implicates a function called window function. It is also known as tapering function. It states that if some interval is chosen, it returns with finite non-zero value inside that interval and zero value outside that interval. A major effect of windowing is that the discontinuities of the frequency response are converted into transition bands between values on either side of the discontinuity.

There are many window techniques available for designing the FIR filter and they are [18][19][20][21]:
1. Hanning window
2. Hamming window
3. Blackman window
4. Rectangular window

5. Bartlett window

6. Kaiser window etc.

\subsection{Hamming Window}

The equation for Hamming window sequence can be defined by [12][13],

$w(n)=\alpha-\beta \cos \left(\frac{2 \pi n}{N-1}\right)$ for $-\frac{N-1}{2} \leq n \leq \frac{N-1}{2}$ (4.1.1)

With $\alpha=0.54$ and $\beta=1-\alpha=0.46$

The non-causal hamming window function is related to the rectangular window function.

$w_{H}(n)=w_{R}(n)\left[0.54+0.46 \cos \frac{2 \pi n}{N-1}\right]$

The spectrum of the hamming window can be obtained as

$$
\begin{aligned}
w_{H}\left(e^{j \omega T}\right)= & 0.54 \frac{\sin \left(\frac{\omega N T}{2}\right)}{\sin \left(\frac{\omega T}{2}\right)}+0.46 \frac{\sin \left(\frac{\omega N T}{2}-\frac{N \pi}{N-1}\right)}{\sin \left(\frac{\omega T}{2}-\frac{\pi}{N-1}\right)}+ \\
& 0.46 \frac{\sin \left(\frac{\omega N T}{2}+\frac{N \pi}{N-1}\right)}{\sin \left(\frac{\omega T}{2}+\frac{\pi}{N-1}\right)} \ldots \ldots \ldots \ldots \ldots \ldots \ldots \ldots \ldots \ldots \ldots \ldots \ldots \ldots . \ldots . . .
\end{aligned}
$$

The width of the main lobe is approximately $8 \pi / \mathrm{N}$ and the peak of the first side lobe is at $-43 \mathrm{~dB}$. The side roll off is 20 $\mathrm{dB} /$ decade. For a causal window,

$$
\begin{aligned}
w_{H}\left(e^{j \omega T}\right)= & 0.54 \frac{\sin \left(\frac{\omega N T}{2}\right)}{\sin \left(\frac{\omega T}{2}\right)}-0.46 \frac{\sin \left(\frac{\omega N T}{2}-\frac{N \pi}{N-1}\right)}{\sin \left(\frac{\omega T}{2}-\frac{\pi}{N-1}\right)}- \\
& 0.46 \frac{\sin \left(\frac{\omega N T}{2}+\frac{N \pi}{N-1}\right)}{\sin \left(\frac{\omega T}{2}+\frac{\pi}{N-1}\right)} \ldots \ldots \ldots \ldots \ldots \ldots \ldots \ldots \ldots \ldots \ldots \ldots \ldots . \ldots . . .
\end{aligned}
$$

\subsection{Hanning Window}

The window function of a causal Hanning window is given by,

$$
w_{\text {Hann }}(n)=\left\{\begin{array}{c}
0.5-0.5 \cos \frac{2 \pi n}{N-1}, 0 \leq n \leq N-1 \\
0, \text { otherwise }
\end{array}\right.
$$

The window function of a non-causal Hanning window is given by,

$$
w_{\text {Hann }}(n)=\left\{\begin{array}{c}
0.5+0.5 \cos \frac{2 \pi n}{N-1}, 0<|n| \leq \frac{N-1}{2} \\
0, \text { otherwise }
\end{array}\right.
$$

The width of the main lobe is approximately $8 \pi / \mathrm{N}$ and the peak of the first side lobe is at $-32 \mathrm{~dB}$.

\subsection{Blackman Window}

The Blackman window sequence can be defined by [1][16][17],

$w_{\text {Black }}(n)=a_{0}+a_{1}+a_{2} \cos \frac{4 \pi n}{N-1}$ for $-\frac{N-1}{2} \leq n \leq \frac{N-1}{2}$

Where $\mathrm{a}_{0}, \mathrm{a}_{1}, \mathrm{a}_{2}$ are constants.

$$
a_{0}=\frac{1-\alpha}{2}, a_{1}=\frac{1}{2}, a_{2}=\alpha / 2
$$

Generally, the value of $\alpha$ for Blackman window is static and it is $\alpha=0.16$. Now, putting the value of $\alpha$ into $a_{0}, a_{1}, a_{2}$, the actual value of the coefficients for the Blackman window 
sequences can be achieved and they are, $\mathrm{a}_{0}=0.42 ; \mathrm{a}_{1}=0.5$; $\mathrm{a}_{2}=0.08$

\section{SIMULATION AND DETERMINA- TION OF WINDOW SEQUENCE AND DESIRED IMPULSE RESPONSE}

In FIR filter, whatever may be the type of the filter, the magnitude response must be 1 at the pass band and otherwise it will be decreased to zero with or without ripple. So, the desired frequency response or in the other hand the magnitude function can de expressed by,

$H_{d}\left(e^{j \omega}\right)=\left\{\begin{array}{c}1 \text { for passband } \\ 0, \text { otherwise }\end{array}\right\}$

The desired impulse response can be derived to,

$h_{d}(n)=\frac{\sin n \pi-\sin \frac{n \pi}{4}}{n \pi}$

If the desired impulse response have the symmetry about $n=0$, it can be obviously said that,

$h_{d}(-n)=h_{d}(n)$

Hence, for a FIR filter of order $\mathrm{N}=6$, the preferred impulse response can be given in the Table-1

Table 1: Impulse Response

\begin{tabular}{|c|c|c|}
\hline $\begin{array}{c}\text { Desired } \\
\text { impulse } \\
\text { Response of } \\
h_{d}(n)\end{array}$ & $\begin{array}{c}\text { Value of } \\
h_{d}(n)\end{array}$ & $\begin{array}{c}\text { Symmetry of } \\
h_{d}(n)\end{array}$ \\
\hline$h_{d}(0)$ & 0.750 & $h_{d}(0)$ \\
\hline$h_{d}(1)$ & -0.225 & $h_{d}(-1)$ \\
\hline$h_{d}(2)$ & -0.159 & $h_{d}(-2)$ \\
\hline$h_{d}(3)$ & -0.075 & $h_{d}(-3)$ \\
\hline$h_{d}(4)$ & 0.000 & $h_{d}(-4)$ \\
\hline$h_{d}(5)$ & 0.045 & $h_{d}(-5)$ \\
\hline
\end{tabular}
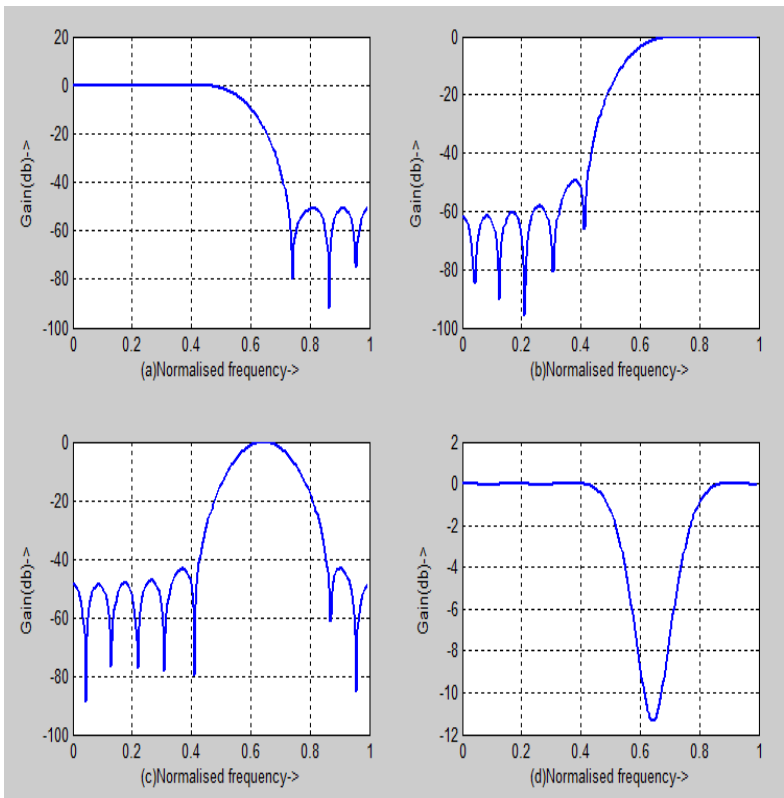

Fig.2: Filters using Hamming window (a) low pass (b) high pass (c) band pass (d) band stop
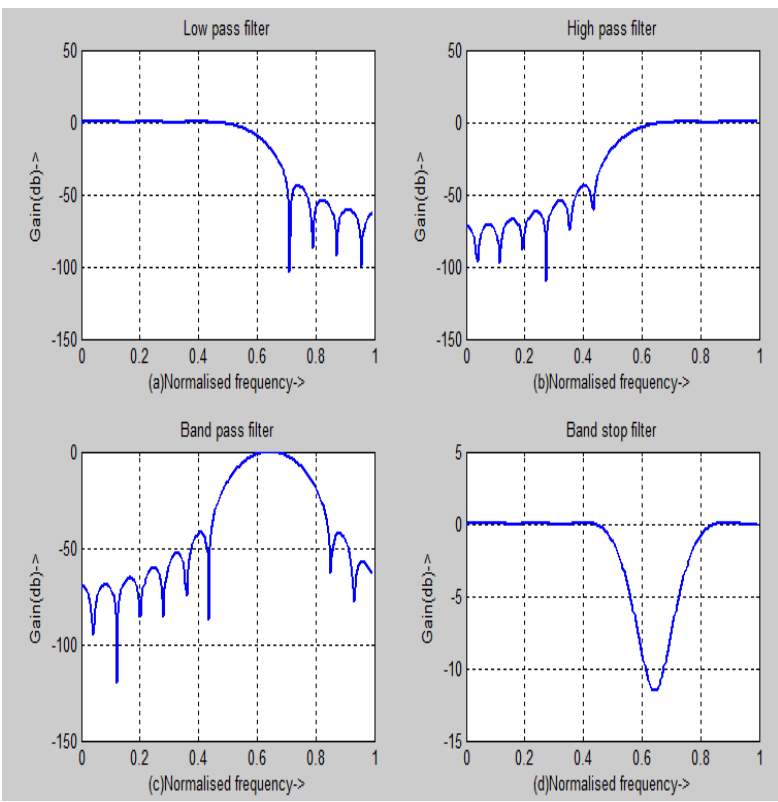

Fig.3: Filters using Hanning window (a) low pass (b) high pass (c) band pass (d) band stop 


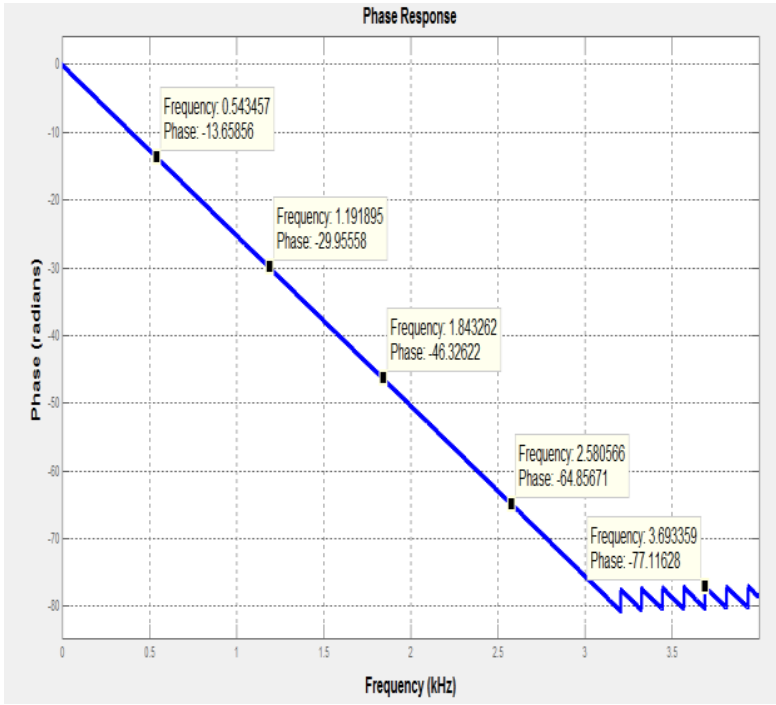

Fig.4: Phase response of Hanning window for low pass filter $(\mathrm{N}=64)$

Table 2: The filter coefficients $h$ (n) using Blackman window sequence

\begin{tabular}{|c|c|c|}
\hline $\begin{array}{c}\text { Filter } \\
\text { coefficients } \\
h(n)\end{array}$ & $\begin{array}{c}\text { Value of } \\
\text { coefficient }\end{array}$ & $\begin{array}{c}\text { Symmetry of } \\
h(n)\end{array}$ \\
\hline$h(0)$ & 0.7500 & $h(0)$ \\
\hline$h(1)$ & -0.2111 & $h(-1)$ \\
\hline$h(2)$ & -0.1229 & $h(-2)$ \\
\hline$h(3)$ & -0.0416 & $h(-3)$ \\
\hline
\end{tabular}

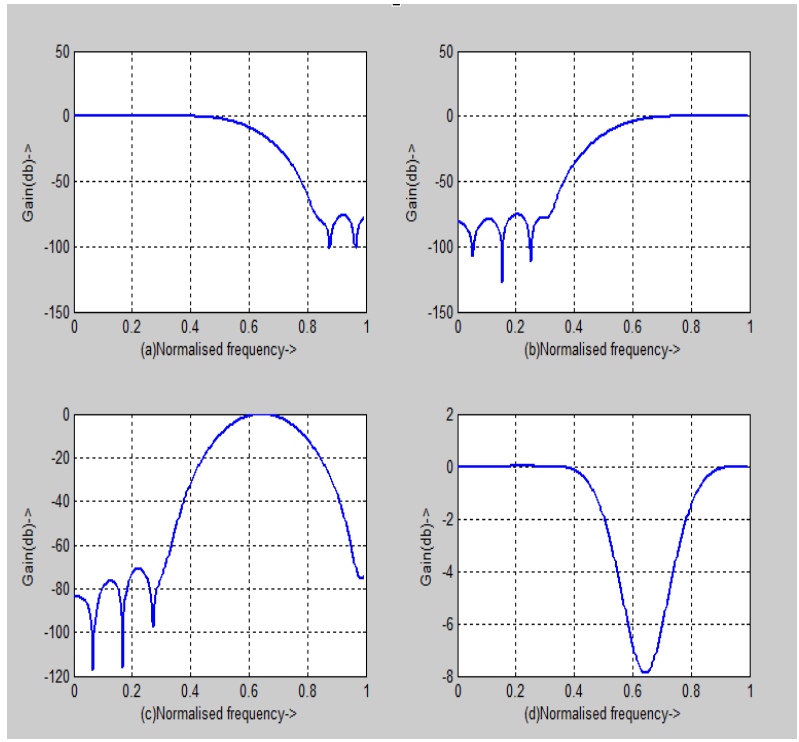

Fig.5: Filters using Blackman window (a) low pass (b) high pass (c) band pass (d) band stop

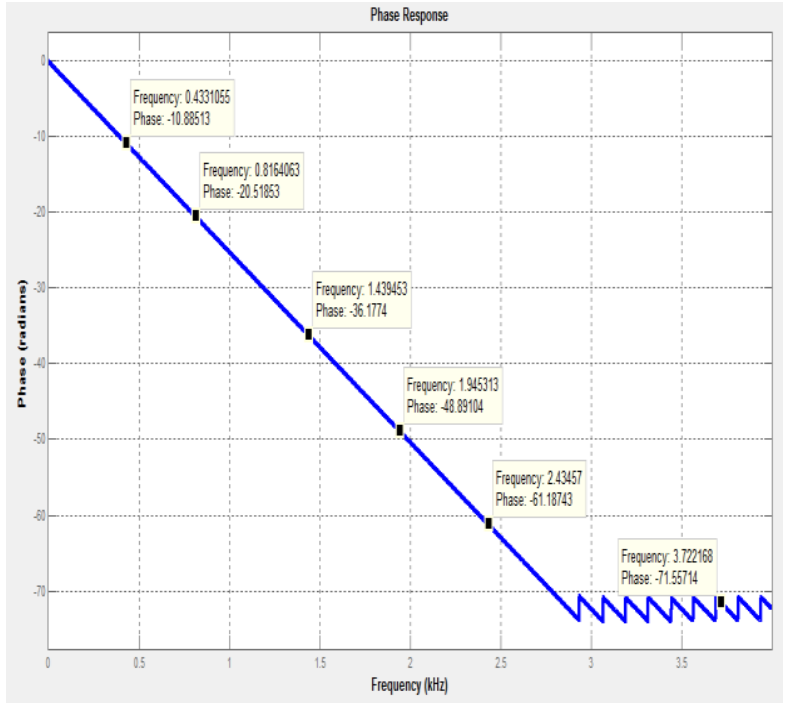

Fig.6: Phase response of Blackman window $(\mathrm{N}=64)$

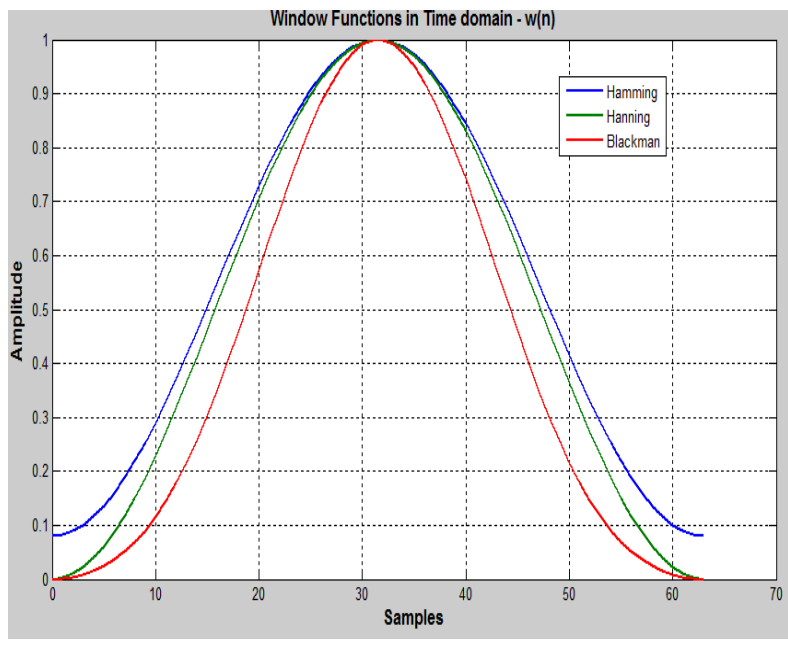

Fig.7: Window function responses in time domain

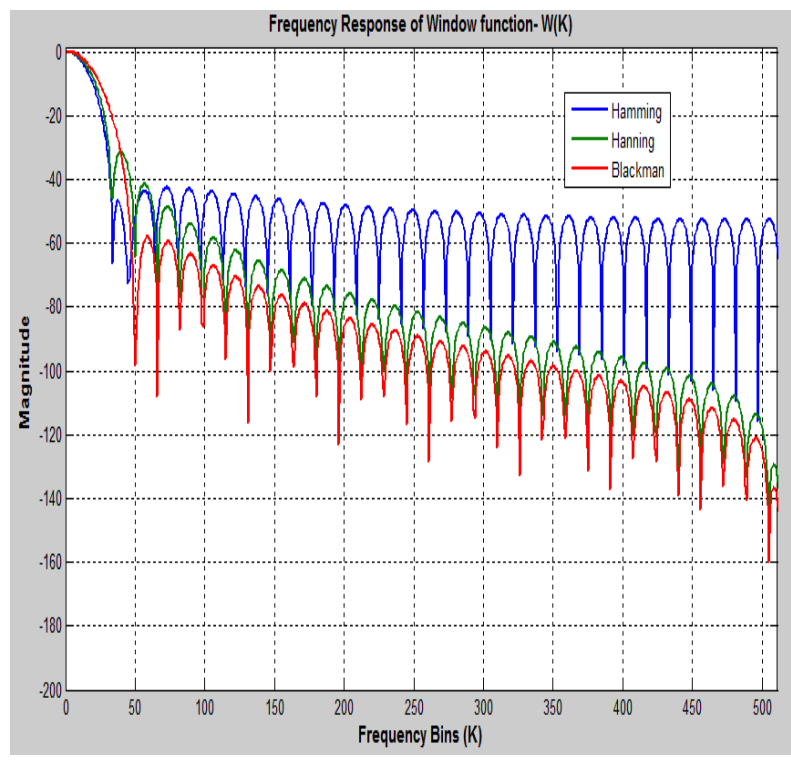

Fig.8: Frequency response of window function 


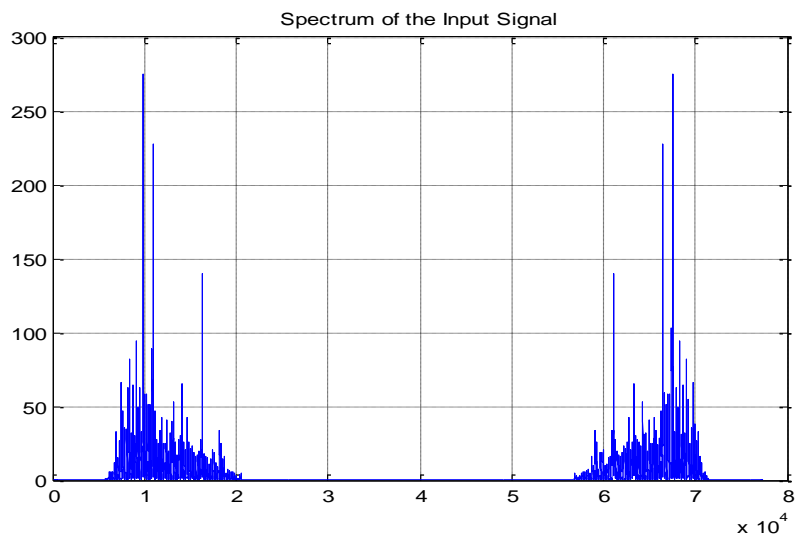

Fig.9: Spectrum of the input signal (speech signal)

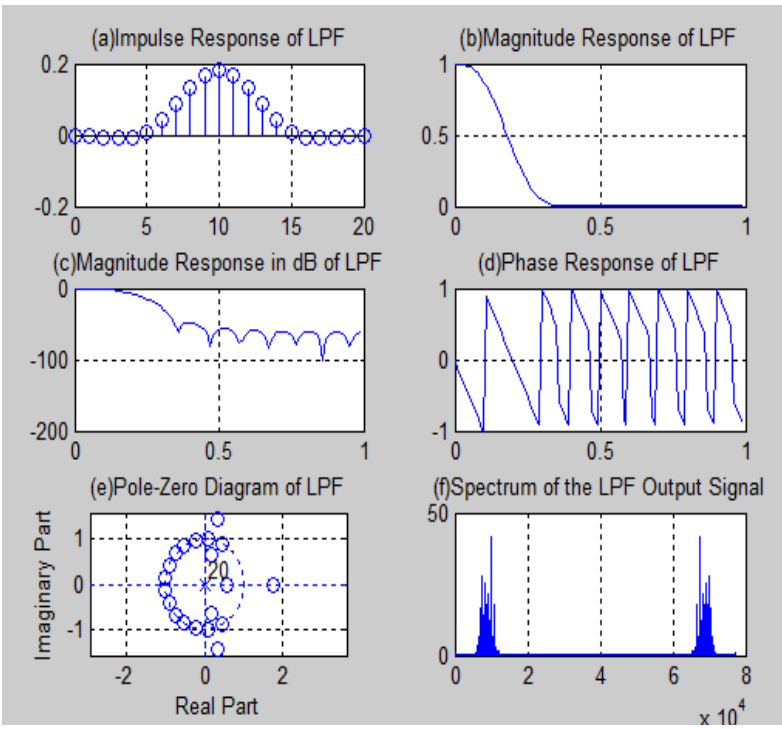

Fig.10: Speech signal analysis for low pass filter using hamming window (order of filter $=20$ )

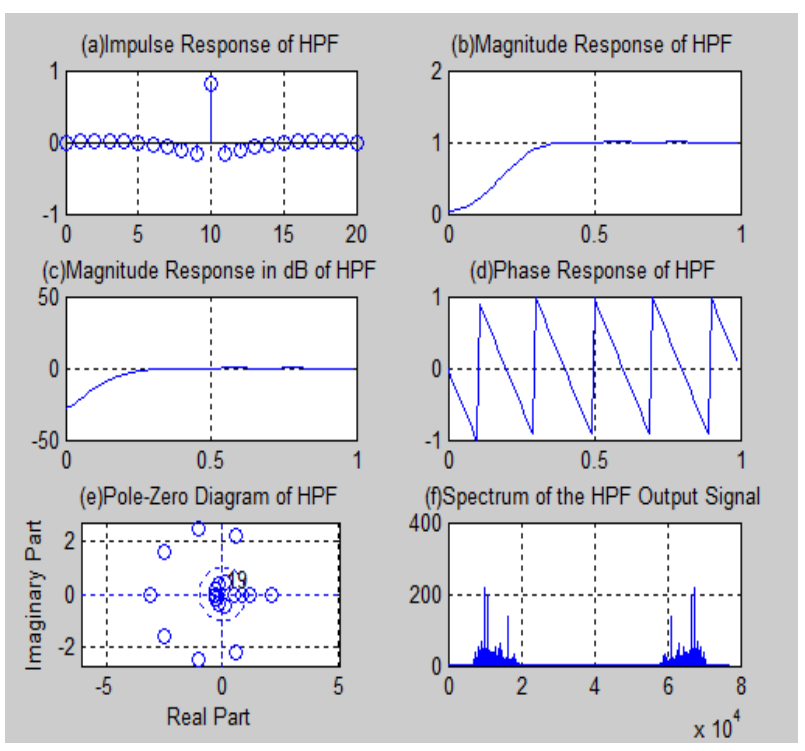

Fig.11: Speech signal analysis for high pass filter using Blackman window (order of filter $=20$ )

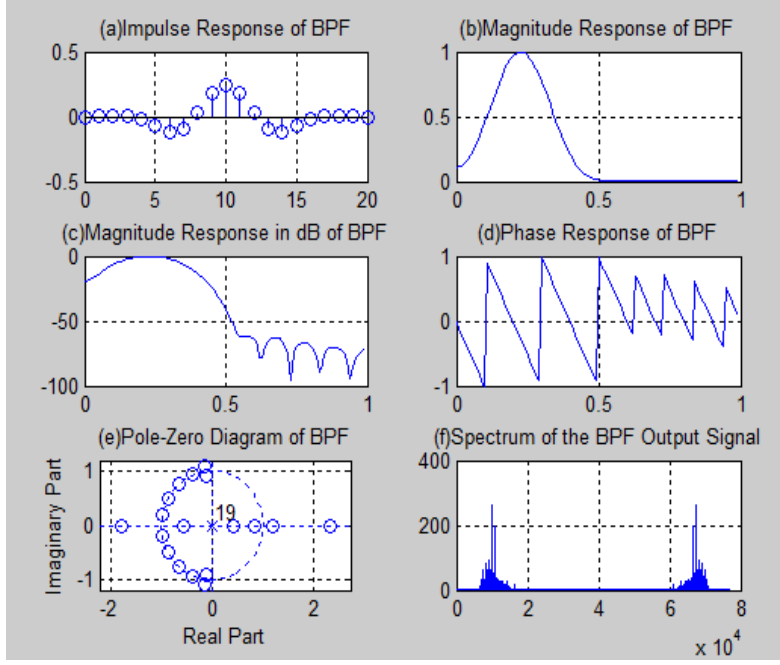

Fig.12: Speech signal analysis for band pass filter using Blackman window (order of filter $=20$ )

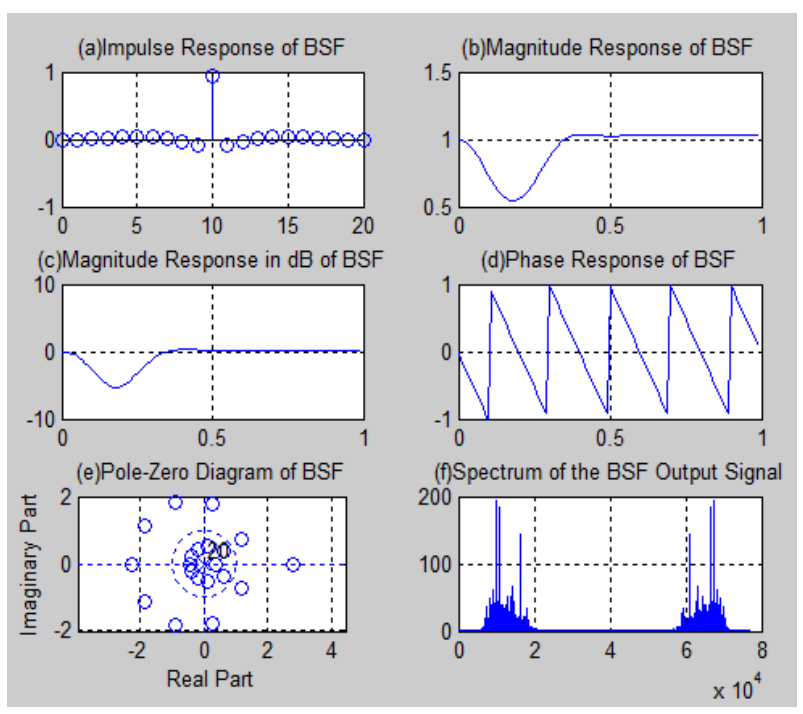

Fig.13: Speech signal analysis for Band stop filter using Hanning window (order of filter=20)

Table 3: ENBW calculations

\begin{tabular}{|c|c|}
\hline $\begin{array}{c}\text { Window } \\
(\mathbf{N}=\mathbf{6 4})\end{array}$ & Equivalent noise bandwidth \\
\hline Hamming & 1.3783 \\
\hline Hanning & 1.5238 \\
\hline Blackman & 1.7542 \\
\hline
\end{tabular}

From above figures (Fig.2, 3, 4, 5 and 6), it can be observed that the response of Blackman window are more smooth and perfect than that of the Hamming and Hanning window. So, the Black man window is more perfect and advantageous than that of those windows. The advantages of Blackman window over Hamming and Hanning window are as follows: 
1. In window sequence function, the Hamming window has fewer terms than compared to the Blackman window. More term in calculation specifies more accuracy in result. For this reason, the simulation result for designing filter is more accurate in Blackman window.

2. There is an extra cosine term in Blackman function. This extra term in terms reduces the side lobes. In Fig.2(a) and Fig.3(a) for FIR low pass filter design, there are many lobes exists in case of Hamming and Hanning window on the other hand for same specification and filter order, in Blackman window, there exists less side lobes in Fig.5(a). Reducing side lobes means that the efficiency is increased. This means less power is lost.

3. The equivalent noise bandwidth is greater in Blackman window than Hanning window (From Table 3) while the ENBW is grater in Hanning window than Hamming window.

4. The response of Blackman window in time domain and frequency domain (Fig.7) is better than Hamming window and Hanning window.

5. In Fig. 2(a), it can be seen that in Hamming window, the peak side lobe is down about 50dB.But in Blackman window, the peak side lobe is down about $78 \mathrm{~dB}$ in fig. 5(a). So, there is a progress in Blackman window of $18 \mathrm{~dB}$ when paralleled to the Hamming window.

Fig.9 shows the spectrum of the speech signal whose sampling rate is 22050 and number of bits per sample is 16 . Fig.10 indicates the low pass filter characteristics and the change of spectrum of the speech signal using Hamming window where the pass band frequency is $2000 \mathrm{~Hz}$. For deigning high pass filter and band pass filter using Blackman window (Fig.11 and Fig.12) pass band frequency $2000 \mathrm{~Hz}$ (for HPF) and pass band and stop band frequency (for BPF) $2000 \mathrm{~Hz}$ and $3000 \mathrm{~Hz}$ have been selected. Fig. 13 shows the band stop filter characteristics and the BSF output spectrum of speech signal using Hanning window where pass band and stop band frequency $1500 \mathrm{~Hz}$ and $2500 \mathrm{~Hz}$ have been used respectively. For speech signal analysis to remove undesirable noise window technique can be used. Band separation filter using Hanning window can recover the original speech signal sharply.

\section{CONCLUSION}

Beside the mathematical comparison among Hamming, Hanning and Blackman window function, in this paper, they have also been encountered with the designed low pass, high pass, band pass and band stop FIR filter with a view to comparing their responses for different parameters like magnitude response, phase response, equivalent noise bandwidth, sidelobe transition width and response in time and frequency domain all done using MATLAB simulation. The filter coefficients are considered for the design of the FIR filter. The equivalent noise bandwidth is also calculated for Hamming and Blackman window. The speech signals have also been encountered using MATLAB simulation, which was the special consideration, and compared the input and output spectrum of the signal. In all cases, it has been found Blackman window showing superiority in performance and demonstrating best functionality among these three windows which is also expected from the theory.

\section{REFERENCES}

[1] Datar A., and Jain A,Sharma, P.C., 2009."Performance of Blackman window famiy in M-channel cosine modulated filter bank for ECG signal", Multimedia, Signal Processing and Communication Technologies, IMPACT '09. International, IEEE Conference, Aligarh, ISBN: 978-1-4244-3602-6, pp 98- 101.

[2] Yu-Chi Tsao and Ken Choi, 2012."Hardware-Efficient Vlsi Implementation For 3-Parallel Linear-Phase Fir Digital Filter Of Odd Length," Circuits and Systems (ISCAS), IEEE Conference, ISBN: 978-1-4673-0218-0, pp. $998-1001$.

[3] Soni, V., and Shukla P.,Kumar M., 2011."Application Of Exponential Window To Design A Digital Nonrecursive Fir Filter", Advanced Communication Technology (ICACT), 13th International Conference, IEEE, ISBN: 978-1-4244-8830-8, pp. 1015-1019.

[4] Mehboob, R., Khan, S.A., and Qamar, R., 2009. "FIR Filter Design Methodology for Hardware Optimized Implementation", Consumer Electronics, IEEE Transactions, Volume: 55, Issue: 3, pp. $1669-1673$.

[5] Sanal, M., Kuloor, R., and Sagayaraj, M.J., 2013. "Optimized FIR filters for digital pulse compression of biphase codes with low sidelobes", Aerospace Conference, IEEE, ISBN: 978-1-4673-1812-9, pp. 1-9.

[6] Saurabh Singh Rajput, and Dr.S.S. Bhadauria, 2012 "Implementation of fir filter using efficient window function and its application in filtering a speech signal," International Journal of Electrical, Electronic and Mechanical Controls, Vol: 1, issue: 1, November.

[7] Ravinder Kaur, Ashish Raman, Hardev Singh and Jagjit Malhotra, 2011. "Design and Implementation of High Speed IIR and FIR Filter using Pipelining", International Journal of Computer Theory and Engineering, Vol. 3, No. 2, ISSN: 1793-8201, pp 292-295.

[8] Proakis, J. G. and Manolakis, D. G. 2007. Digital Signal Processing: Principles, Algorithms, and Applications. Pearson Education Ltd.

[9] S. M. Shamsul Alam, Md. Tariq Hasan, "Performance Analysis of FIR Filter Design by Using Optimal, Blackman Window and Frequency Sampling Methods", International Journal of Electrical \& Computer Sciences (IJECS) Vol: 10, pp.13-18.

[10] Sarita Chouhan, and Yogesh Kumar, 2012. "Low power designing of FIR filters," International Journal of Advanced Technology \& Engineering Research, ISSN: 2250-3536 Volume 2, Issue 2, pp. 59-67.

[11] Magatha Nayak Bhukya, K. Anjaiah, G. Sravya, P. Nagaraju, 2012 "The Design of High Speed FIR Filter using Improved DA Algorithm and it's FPGA Implementation," International Journal of Engineering Trends and Technology,Vol:3, , pp. 123-126.

[12] Manoj Garg, Rakesh Kumar Bansal, and Savina Bansal, "Reducing Power Dissipation in FIR Filter: An Analysis," Signal Processing: An International Journal (SPIJ), Vol: 4, pp. 62-67.

[13] Gerard Blanchet and Maurice Charbit, 2006, "Digital Signal and Image Processing using Matlab", ISTE Ltd., (C) HERMES Science Europe Ltd, 2001, (C) ISTE Ltd, ISBN-13: 978-1-905209-13-2,ISBN-10: 1-905209-13-4

[14] J.S. Chitode, "Digital Signal Processing", Technical Publication, Pune, ISBN: 9788184314243. 
[15] Dag Stranneby, "Digital Signal Processing-DSP \& Application", Butterworth-Heinemann, Oxford, ISBN: 0750648112, 2001.

[16] Michael Weeks, "Digital Signal Processing Using MATLAB and Wavelets", Infinity Science Press, Hingham, Massachusetts, ISBN: 0-9778582-0-0, 2007.

[17] Taan S. ElAli, "Discrete Systems and Digital Signal Processing with Matlab," CRC Press, ISBN 0-203487117, 2004.

[18] Bob Meddins, "Introduction to Digital Signal Processing", Essential Electronics Series, Newnes, Butterworth-Heinemann, Oxford, ISBN: 0750650486, 2000.

[19] Ramesh .R, and Nathiya .R, 2012. "Realization of fir filter using modified distributed arithmetic architecture", Signal \& Image Processing: An International Journal (SIPIJ) Vol.3, No.1, pp 83-94.

[20] P. Ramesh Babu,'Digital Signal Processing", Fourth edition, Scitech Publication(India) Pvt. Ltd, Chennai,2008.

[21] Andreas Antoniou, "Digital Signal Processing: Signals, Systems and Filters", Tata McGraw-Hill Education, ISBN-10: 0070636338, 2006.

[22] Taan S. ElAli, "Discrete Systems and Digital Signal Processing with Matlab", CRC Press, ISBN 0-203487117, 2004.

\section{AUTHORS' PROFILE}

Prajoy Podder is currently pursuing B.Sc. degree program in Electronics and Communication Engineering in Khulna
University of Engineering \& Technology, Khulna-9203, Bangladesh. His research interest includes antenna design, machine learning, pattern recognition, neural networks, computer networking, VLSI system design, image processing, telecommunication and feature selection.

Tanvir Zaman Khan is currently pursuing B.Sc. degree program in Electronics and Communication Engineering in Khulna University of Engineering \& Technology, Khulna9203, Bangladesh. His research interest includes antenna design, machine learning, pattern recognition, neural networks, computer networking, VLSI system design, image processing, embedded system design, web security \& application, telecommunication and feature selection.

Mamdudul Haque Khan is currently pursuing B.Sc. degree program in Electronics and Communication Engineering in Khulna University of Engineering \& Technology, Khulna9203, Bangladesh. His research interest includes antenna design, machine learning, pattern recognition, neural networks, computer networking, VLSI system design, image processing, embedded system design, web security \& application, telecommunication and feature selection.

M. Muktadir Rahman is currently pursuing B.Sc. degree program in Electronics and Communication Engineering in Khulna University of Engineering \& Technology, Khulna9203, Bangladesh. His research interest includes antenna design, machine learning, pattern recognition, neural networks, computer networking, VLSI system design, image processing, embedded system design, web security \& application, telecommunication and feature selection. 